\title{
Journalistische Verantwortungsethik und sexueller Missbrauch
}

\author{
Eine Untersuchung der Perspektive von Betroffenen auf Journalist*innen und deren \\ Berichterstattung
}

\section{Philip Baugut / Katharina Neumann}

Sexueller Missbrauch ist ein häufig verborgener Missstand, der gerade dann ins öffentliche Bewusstsein rücken kann, wenn Betroffene den Weg in die Medienöffentlichkeit wäblen. Um zu ergründen, welche Verantwortung Journalist*innen dabei gegenüber Betroffenen tragen, untersucht diese Studie die weitgehend unerforschten Medienwirkungen auf Betroffene, die im Zentrum von Berichterstattung standen. Vor dem theoretischen Hintergrund von Kepplingers Konzept der reziproken Effekte zeigten 18 qualitative Interviews mit Betroffenen, dass diese schweren Belastungen ausgesetzt sind, wenn Journalist*innen einer am Primat der Publikumsaufmerksamkeit orientierten Medienlogik folgen und das politische Anliegen der Betroffenen ignorieren, vor allem die strukturellen Ursachen sexuellen Missbrauchs zu thematisieren. Bestimmte als respektlos empfundene journalistische Verhaltensweisen und Berichterstattungsmuster können für Betroffene bedeutende psychische Folgen haben, auch weil sie relativ starke Medienwirkungen auf Dritte wabrnebmen. Insgesamt zeigen die Betroffenen ein differenziertes Medienbild, das von Dankbarkeit für lang ersebntes öffentliches Gebör bis hin zu „Voyeurismus“-Vorwürfen an Journalist*innen reicht.

Schlüsselwörter: Medienwirkungen; reziproke Effekte; sexueller Missbrauch; Verantwortungsethik

\section{Einleitung}

Gelegentlich werde ich gefragt, warum ich mich als Missbrauchsopfer geoutet habe. Dieses Wort gefällt mir gar nicht: outen. Denn es bedeutet, dass man öffentlich eine Peinlichkeit zugibt. Der Missbrauch ist aber nur peinlich für den Täter. Ich bin das Opfer und sehe den Schritt in die Öffentlichkeit als Akt der Gerechtigkeit.

(Betroffener sexuellen Missbrauchs, zitiert in ZEIT ONLINE 2019)

Sexueller Kindesmissbrauch (SKM) ist ein globales Problem. Jedes achte Kind auf der Welt ist betroffen (Mathews \& Collin-Vézina 2016), laut Experten leben allein in Deutschland aktuell eine Million Kinder, die sexuell missbraucht wurden (Rörig 2017). SKM findet nicht nur im familiären Umfeld, im Bekanntenkreis, in Schulen oder Sportvereinen statt, sondern auch innerhalb der Kirche (Dreßing et al. 2019; Terry \& Jay 2015). Im Jahr 2018 legte eine von der Deutschen Bischofskonferenz in Auftrag gegebene Studie offen, dass 3677 Kinder und Jugendliche in den vergangenen Jahrzehnten von Kirchenvertretern in Deutschland missbraucht wurden (Dreßing et al. 2018). Das Dunkelfeld wird auf weit über 100.000 Opfer geschätzt (Witt et al. 2019).

Eine intensive öffentliche Debatte über SKM entzündete sich in Deutschland im Jahr 2010, als bekannt wurde, dass einige Schüler des katholischen Berliner „Canisius-Kollegs“ über Jahre hinweg von ihren Lehrern missbraucht wurden (Füller 2011). Nach Aufdeckung dieses Skandals erfuhr die Öffentlichkeit in den folgenden Jahren von weiteren Missbrauchsfällen, das lange tabuisierte Thema „sexueller Kindesmissbrauch“ erhielt schließlich intensivere Medienaufmerksamkeit (ebd.). Zahlreiche Betroffene sind 
mit ihrer persönlichen Geschichte in die Öffentlichkeit gegangen - obgleich es diesen häufig schwerfällt, das Erlebte gegenüber Dritten zu verbalisieren (Kavemann et al. 2016: 27). Dies birgt die Gefahr, dass ein gravierendes Problem nicht die (Medien-)Öffentlichkeit erhält, die es braucht, gerade auch unter Präventionsgesichtspunkten. Daher erscheint die Perspektive der Betroffenen auf Journalist*innen und deren Berichterstattung als wichtiges Untersuchungsobjekt.

Bislang wurde kaum systematisch untersucht, warum Betroffene (Medien-)Öffentlichkeit suchen, wie ihr Kontakt mit Journalist*innen abläuft, wie sie die daraus resultierende Berichterstattung wahrnehmen und wie diese auf sie wirkt. Systematisch gewonnene Erkenntnisse darüber, wie Betroffene Journalist*innen und deren Berichterstattung über ihren Fall wahrnehmen, sollten in mehrerer Hinsicht Praxisrelevanz entfalten.

Erstens sollten sie Journalist*innen helfen, sich gegenüber den Betroffenen verantwortlich zu verhalten, gerade mit Blick auf deren psychisches Wohlbefinden. Entsprechend fokussiert Thomaß‘ (2003) Vorschlag für „fünf ethische Prinzipien journalistischer Praxis“ nicht nur die Beziehung der Journalist*innen zu ihren Quellen, den Rezipienten, den Kollegen und der allgemeinen Öffentlichkeit, sondern eben auch die Beziehung zu den Berichterstattungsobjekten, die vom Persönlichkeitsschutz geprägt sein sollte. Grundsätzlich ist die mediale Darstellung realer Gewalt, worunter sexueller Missbrauch fallen kann, ein zentrales Thema der kommunikationswissenschaftlichen Teildisziplin Medienethik (z. B. Bohrmann 2010). Deren kritische Reflexion journalistischen Handelns erscheint gerade auch deshalb notwendig, weil ökonomische Imperative, die sich aus Medienwettbewerb und Profitorientierung ergeben (Brosda 2010), dazu verleiten können, diese über das Prinzip der Achtung gegenüber den Objekten der Berichterstattung zu stellen. In der letzten großen Repräsentativ-Befragung von Journalist*innen in Deutschland gaben mehr als drei Viertel der Befragten an, "Journalismusethik“ sei „extrem oder sehr einflussreich“ auf ihre Arbeit - über Profiterwartungen sagte dies nur etwa jede*r Fünfte (Hanitzsch, Steindl \& Lauerer 2016). Inwieweit sich diese (Eigen-)Wahrnehmungen damit decken, wie Betroffene Journalist*innen erleben, gilt es herauszufinden.

Zweitens kann kommunikationswissenschaftliches Wissen darüber, unter welchen Umständen der Gang an die Öffentlichkeit für Betroffene hilfreich oder gar gefährlich ist, für die psychotherapeutische Praxis von Nutzen sein. Drittens lässt sich anhand des Beispiels der sexuellen Missbrauchsthematik möglicherweise grundsätzlicher verstehen, wo die Logik journalistischer Medien, die um Publikumsaufmerksamkeit kämpfen, an ethische Grenzen stößt, weil nicht jeder Gegenstand der Berichterstattung denselben universellen journalistischen Routinen unterliegen kann.

Vor diesem Hintergrund wurde die zentrale Forschungsfrage, welche Perspektive Betroffene auf journalistische Medien haben, anhand von qualitativen Leitfadeninterviews mit Personen untersucht, die den Weg in die Medienöffentlichkeit gewählt haben - und somit über einschlägige medienbezogene Erfahrungen berichten konnten. Obgleich sich die Täter in unterschiedlichen gesellschaftlichen Bereichen finden, konzentriert sich diese Studie auf die Opfer von Tätern innerhalb der Kirche. Dafür sprechen die bereits erwähnten Zahlen, die wohl auch deshalb zu intensiverer Medienberichterstattung über die Betroffenen geführt haben, weil Missbrauch innerhalb der Strukturen einer der größten gesellschaftlichen Institutionen, nämlich der Kirche, eine besondere politische Dimension besitzt. 


\section{Sexueller Missbrauch und Medienöffentlichkeit}

Den kommunikationswissenschaftlichen Forschungsstand prägen Studien zur Darstellung sexueller Gewalt in den Medien (Hove et al. 2013; Maydell 2018; Niner, Ahmad \& Cuthbert 2013; Popović 2018; Scheufele 2005; Weatherred 2015; für eine Zusammenfassung des Forschungsstands: Döring 2018). Zu den zentralen Kritikpunkten an der Berichterstattung gehört deren Tendenz zur Stereotypisierung: Täter erscheinen als fremdartige "Monster“ (Cromer \& Goldsmith 2010; Döring 2018), Opfer als bemitleidenswerte, psychisch dauerhaft gebrochene Personen (Döring 2018; Hölling 2007). Darüber hinaus konzentriert sich die Berichterstattung auf schwere physische Gewalt, während die langfristigen psychologischen Folgen von Missbrauch in den Hintergrund rücken (Scheufele 2005). Häufig betont wird die Notwendigkeit, Täter zu bestrafen (ebd.), seltener wird hingegen über Präventionsmaßnahmen berichtet (Döring 2018; Mejia, Cheyne \& Dorfman 2012; Waller et al. 2020) oder auf Hilfsangebote hingewiesen - was sich Betroffene jedoch von Berichten über Missbrauch wünschen würden (Kavemann et al. 2018).

Genau diese Betroffenen-Perspektive ist bislang relativ unterbelichtet geblieben, im Vordergrund standen Wirkungen auf das breite Publikum (z. B. Kitzinger 2004; Scheufele 2005). Zur Schließung dieser wichtigen Forschungslücke haben Studien beigetragen, die zeigen, dass Betroffene sexuellen Missbrauchs an der entsprechenden Berichterstattung zum Beispiel deren mangelnde Intensität kritisieren (Döring \& Walter 2019). Auch würden die Betroffenen nicht ausreichend in den Medien zu Wort kommen, die Berichterstattung sei häufig zu „reißerisch“ aufbereitet, sie würde zu stark auf Tatdetails bzw. Täter fokussieren und stigmatisierend wirken (z. B. Hölling 2007; Kaveman et al. 2018; Kunczik \& Bleh 1995).

Generell reflektiert die Kritik der Betroffenen an der Berichterstattung das, was journalistische Leitlinien und wissenschaftliche Literatur für wichtige Qualitätskriterien der Berichterstattung halten (Döring \& Walter 2019): (a) Berichterstattung sollte sich nicht auf Einzelfälle fokussieren, sondern diese als generelles, gesellschaftliches Problem darstellen. (b) Es sollte nicht sensationalistisch berichtet werden. (c) Irreführende oder verharmlosende Begrifflichkeiten gilt es zu vermeiden. (d) Stakeholder wie Beratungsstellen sollten zu Wort kommen. (e) Auf irreführende Stereotype bzw. Mythen ist zu verzichten. (f) Möglichkeiten zur Prävention und Intervention im Sinne einer Unterstützung der Opfer sind zu thematisieren. (g) Opfern sollte in der Interviewsituation respektvoll und sensibel begegnet werden. (h) Die Berichterstattung hat gesetzeskonform zu sein, dabei spielt der Persönlichkeitsschutz der Opfer eine wichtige Rolle. ${ }^{1}$

Es ist jedoch fraglich, inwieweit die genannten Befunde die Perspektive derjenigen Betroffenen widerspiegeln, die mit ihren Fällen tatsächlich Gegenstand von Berichterstattung waren. Zwar liegt nahe, dass sich Betroffene stark mit anderen Betroffenen identifizieren und daher Berichterstattung über sexuellen Missbrauch generell intensiv wahrnehmen. Mit der eigenen Geschichte in den Medien präsent zu sein und persönliche Erfahrungen im Umgang mit Journalist*innen zu machen, könnte jedoch mit einer spezifischen Sicht auf die Medien verbunden sein. Die Perspektive von Personen, die als Fallbeispiele in der Berichterstattung auftauchen, markiert somit eine Forschungslücke, die es auch deshalb zu schließen gilt, weil Berichterstattung über das, was Betroffene

1 Darüber hinaus haben Döring und Walter (2019) deduktiv aus der Literatur zwei weitere Qualitätskriterien im Umgang mit der Thematik hergeleitet: die Ausgewogenheit der Interessen von Opfern und potenziellen Tätern (Unparteilichkeit) sowie die Offenlegung und Reflexion von Quellen (Transparenz). 
erlebt haben, für diese bedeutsame psychische Folgen haben könnte. Eine quantitative Befragung von Kriminalitätsopfern aus dem Jahr 1995 legt nahe, dass sowohl die Merkmale der medialen Darstellung als auch die Reaktionen Dritter darüber entscheiden können, wie Medienberichte den psychologischen Zustand von Opfern beeinflussen. So liefert die Literatur auch Hinweise darauf, dass beispielsweise eine zu detaillierte Darstellung des konkreten Tathergangs (z. B. Johnson 2017; Martinius 2009) negative Emotionen wie Scham hervorrufen kann. Auch wenn Missbrauchsopfer mangelnde Kontrolle über die eigene mediale Darstellung spüren, könnte dies problematische psychische Folgen haben. So zeigt die Forschung, dass gerade traumatisierte Betroffene sexueller Gewalt mit Ohnmachtsgefühlen zu kämpfen haben (z. B. Herman 2018). Als zentrales Ziel der Behandlung von Traumata, wie sie infolge sexuellen Missbrauchs auftreten können, gilt entsprechend, dem Patienten Wege aus seiner erlebten Hilflosigkeit aufzuzeigen und ihm das Gefühl zu geben, Wirkungsmacht zu besitzen (ebd.). Bedeutsam erscheint daher auch die Frage, welches journalistische Handeln bei Betroffenen unter Umständen ein Gefühl von Kontrollverlust auslösen kann (Fritsche, Jonas \& Kessler 2011).

\section{Ein Modell reziproker Effekte auf Betroffene sexuellen Missbrauchs}

Um die vorliegende Studie theoretisch zu fundieren, wird auf das Konzept reziproker Effekte (Lang \& Lang 1953; Kepplinger 2007, 2010) zurückgegriffen. Dieses beschreibt eine Reihe spezifischer Medienwirkungen, die Personen im Zentrum der Berichterstattung zeigen, wenn sie Medienberichte über sich selbst rezipieren. Kepplinger (2007; 2010) unterscheidet in diesem Zusammenhang zwischen dem breiten Publikum, sog. bystanders, und den Protagonisten der Berichterstattung, über die ad personam berichtet wird. Anders als von der Berichterstattung unbeteiligte bystanders zeigen Protagonisten infolge ihrer persönlichen Betroffenheit besonders starke Reaktionen auf Medienberichte. Die kommunikationswissenschaftliche Beschäftigung mit reziproken Effekten hat sich in den letzten Jahren zwar intensiviert (z. B. Daschmann 2007; Kepplinger \& Glaab 2005; Kepplinger \& Marx 2008), allerdings behandelten bisherige Studien vor allem Medienwirkungen auf Entscheidungsträger wie Politiker (Kepplinger \& Marx 2008) oder Richter und Staatsanwälte (Kepplinger \& Zerback 2009). Gewaltopfer wurden bislang noch nicht aus der theoretischen Perspektive des Konzepts reziproker Effekte unter die Lupe genommen.

Vor diesem Hintergrund wird in Anlehnung an Kepplingers Modell reziproker Effekte (2010) zunächst ein theoretisches Gerüst entwickelt, das die Perspektive von Betroffenen sexuellen Missbrauchs auf Journalist*innen und deren Berichterstattung systematisieren soll.

\section{Modellblock 1: Kontakt zu Journalist*innen}

Zunächst stellt sich die Frage nach dem Ausgangspunkt der Berichterstattung über einzelne Fälle sexuellen Missbrauchs. Anders als bei (prominenten) Personen von öffentlichem Interesse, die wie Politiker stets damit rechnen müssen, dass gegen ihren Willen über sie berichtet wird (Daschmann 2007; Kepplinger 2009), ist bei Betroffenen von sexuellem Missbrauch damit zu rechnen, dass über sie persönlich nur mit ihrem Einverständnis berichtet wird. Dies führt zur Frage nach der Motivation der Betroffenen, ihren Fall öffentlich zu machen und hierfür mit Journalist*innen zu interagieren. Frühere Studien (z. B. Kavemann et al. 2016) haben gezeigt, dass Betroffene, die sich Vertrauens- 
personen öffnen, beispielsweise darauf hoffen, Zugang zu professioneller Unterstützung bei der Verarbeitung des Erlebten zu erhalten (Kavemann et al. 2016: 109f.).

Die Interaktion von Betroffenen mit Journalist*innen erscheint als eine für beide Seiten komplexe Situation: So gilt es für Journalist*innen, mit (in vielen Fällen) traumatisierten Personen über eine Thematik zu sprechen, die selbst Psychotherapeuten vor größere Herausforderungen stellt (Kavemann et al. 2016). Im professionellen Korsett, das Journalist*innen Publikumsorientierung bzw. Publikumsaufmerksamkeit versprechende Beiträge abverlangt (z. B. Meyen \& Riesmeyer 2009), werden traumatisierte Betroffene möglicherweise zu unangenehmen Details befragt (Müller \& Nicolai 2007), was wiederum Folgen für ihr psychisches Wohlbefinden und ihre damit verbundene Auskunftsbereitschaft haben kann. Umgekehrt ist es allerdings ebenso denkbar, dass ein von den Journalist*innen mit Empathie geführtes Interview von Betroffenen als Beitrag zur Bewältigung des Erlebten wahrgenommen wird - auch weil der Versuch gelingt, sich einer Person außerhalb des familiären bzw. therapeutischen Umfeldes anzuvertrauen.

Vor diesem Hintergrund stellen sich für den Modellblock 1 die folgenden Forschungsfragen:

\subsection{Warum entscheiden sich Betroffene sexuellen Missbrauchs dazu, mit Journalist*innen öfentlich über ibren Fall zu sprechen?}

\section{2 Über welche Wege findet der Kontakt mit Journalist*innen statt?}

\subsection{Wie nebmen sie die Interaktion mit Journalist*innen wabr?}

\section{Modellblock 2: Medienverarbeitung}

Kommt es zur Berichterstattung über einzelne Missbrauchsfälle, ist mit Blick auf die Frage nach deren Wirkungen auf die Betroffenen zunächst zu klären, wie diese die Berichterstattung verarbeiten. Prinzipiell können Protagonisten der Berichterstattung anders als das unbeteiligte Publikum - Medienberichte mit ihren eigenen Erfahrungen abgleichen. Dies führt immer wieder dazu, dass sich die Protagonisten falsch dargestellt und von Journalist*innen unfair behandelt fühlen (Daschmann 2007; Kepplinger 2010). Gerade bei Betroffenen sexuellen Missbrauchs könnte dies der Fall sein, gerade wenn ihnen traumatische Erfahrungen stets präsent sind. Auch könnten Betroffene infolge einer tief verankerten negativen Einstellung gegenüber der Institution Kirche den Tenor der Berichterstattung über die Kirche bzw. Täterseite verzerrt wahrnehmen (Vallone, Ross \& Lepper 1985) und dadurch negativer bewerten.

Neben der Bewertung der Berichterstattung erscheint grundsätzlich auch bedeutsam, welche Annahmen die Betroffenen über Medienwirkungen auf Dritte (Davison 1983) bzw. die öffentliche Meinung (Gunther 1998) haben. Im Sinne der Logik des „Influenceof-Presumed-Media-Influence“-Ansatzes (Gunther \& Storey 2003) könnte etwa die Annahme, dass eine bestimmte Form der Veröffentlichung des eigenen Falls potenzielle weitere Täter abschreckt, dazu führen, dass Betroffene eher bereit sind, (weiterhin) Medienöffentlichkeit zu suchen. Umgekehrt könnte die Annahme, dass das eigene soziale Umfeld auf die Berichterstattung negativ reagiert, beispielsweise zu Angst und einer geringeren Bereitschaft führen, offen mit Journalist*innen zu sprechen. Damit gewinnt die Frage an Relevanz, welche Verhaltensänderungen Betroffene nach der Veröffentlichung ihres Falls in ihrem sozialen Umfeld wahrnehmen (Kepplinger 2010). Bekannt ist, dass die Reaktionen Dritter maßgeblich darüber entscheiden, ob es Missbrauchsopfern hilft oder schadet, wenn sie sich jemandem anvertrauen (ebd.: 114; Hinkelman \& Bruno 2008). Gerade wenn Betroffene im Medienfokus stehen, sind sie vermutlich unterschied- 
lichen Reaktionen verschiedener Personen(-gruppen) ausgesetzt, die den psychologischen Verarbeitungsprozess des Erlebten maßgeblich beeinflussen - darauf deuten in anderen Kontexten durchgeführte Studien zu Medienwirkungen auf Protagonisten der Berichterstattung hin (z. B. Kepplinger \& Glaab 2005; Kepplinger \& Marx 2008).

Selbst wenn negative Reaktionen Dritter möglicherweise gar nicht ursächlich aus der Rezeption von Medienberichten hervorgehen, erscheint es möglich, dass Betroffene sexuellen Missbrauchs diese wahrgenommenen Reaktionen auf Medienberichte zurückführen, also eine Attribution vornehmen (Allport 1954), infolge derer sie ihren Gang in die Öffentlichkeit bereuen. Vor diesem Hintergrund enthält Modellblock 2 folgende Forschungsfragen:

2. Wie bewerten Betroffene sexuellen Missbrauchs Medienberichte über ibren Fall und welche Medienwirkungen auf Dritte nebmen sie in diesem Zusammenhang an?

\section{Modellblock 3: Medienwirkungen}

Im Bereich der Medienwirkungen sollen sowohl Emotionen und Kognitionen als auch Verhaltenswirkungen berücksichtigt werden (Kepplinger 2010). Im Sinne appraisaltheoretischer Ansätze gelten Emotionen und Kognitionen, zu denen etwa Einstellungen und Realitätsvorstellungen gehören, als voneinander wechselseitig abhängig (Daschmann 2007). Besonders relevant erscheint im Untersuchungskontext die Frage, inwiefern Medienberichte Kognitionen und Emotionen beeinflussen, die für den psychischen $\mathrm{Zu}-$ stand der Betroffenen von Bedeutung sind. Im Bereich der Verhaltenswirkungen wird mit Kepplinger (2010) auf die Frage fokussiert, was Betroffene tun, um eine als falsch wahrgenommene Darstellung zu korrigieren oder eine als wünschenswert wahrgenommene Darstellung erneut zu erzielen. So gesehen können bestimmte Medienwirkungen nicht nur Folge, sondern im Sinne der Reziprozität auch Ursache bestimmter Kontakte $\mathrm{zu}$ Journalist*innen sein (siehe Abb. 1).

Vor diesem Hintergrund umfasst Modellblock 3 folgende Forschungsfrage:

3. Welche Wirkungen haben Medienberichte über Betroffene sexuellen Missbrauchs auf deren Emotionen, Kognitionen und Verbalten?

\section{Abbildung: Ein Modell reziproker Effekte auf Betroffene sexuellen Missbrauchs}

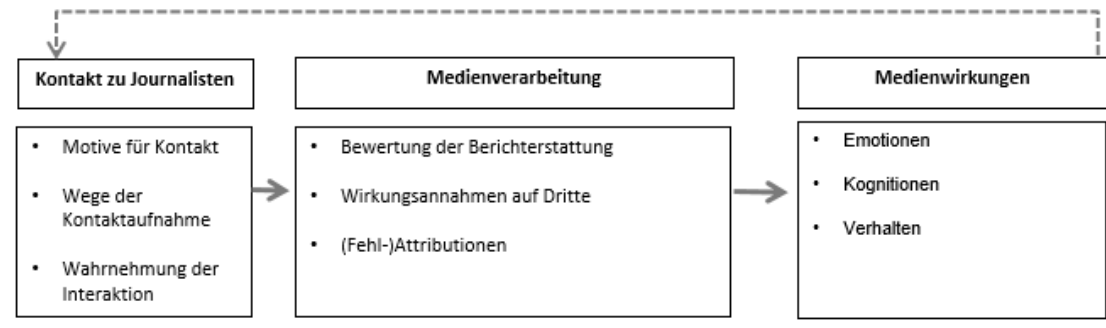

Eigene Darstellung 


\section{Methode}

Um die Perspektive Betroffener auf Journalist*innen und deren Berichterstattung zu untersuchen, wurden 18 qualitative Interviews mit Betroffenen geführt, die in ihrer Kindheit bzw. Jugend von Kirchenvertretern sexuell missbraucht wurden. Alle Befragten waren mit ihren Fällen vor dem Zeitpunkt der Befragung bereits Gegenstand von Berichterstattung. Zur Rekrutierung dieses speziellen Personenkreises wurde im Rahmen einer Internetrecherche mit verschiedenen Suchbegriffen (z. B. „Missbrauch“ und „Kirche“) nach in deutschen Massenmedien erschienenen journalistischen Beiträgen in textlicher und/oder audiovisueller Form gesucht (z. B. Talksendungen zum Thema Missbrauch). Dabei zeigte sich, dass die Zahl der Betroffenen, die mit ihrem Fall in den journalistischen Medien präsent waren, offenbar überschaubar ist. Es konnten jedoch 18 Personen identifiziert werden, deren Mail-Adressen sich recherchieren ließen - beispielsweise, weil sie in Opfervereinen oder anderen Initiativen zum Thema „sexueller Missbrauch“"waren und ihre Kontaktdaten über die entsprechenden Internetseiten öffentlich verfügbar waren. Zusätzlich vermittelten uns unsere Interviewpartner weitere Kontakte zu Betroffenen mit Medienerfahrungen. In diesen Fällen wurde unsere Anfrage zunächst von unseren Interviewpartnern an die jeweiligen Personen weitergeleitet, die dann bei Interesse an einem Interview telefonisch oder per Mail Kontakt mit uns aufnahmen.

Mit 18 Personen kam schließlich ein persönliches oder telefonisches Interview zustande. Diese 18 Personen waren in unterschiedlichen Mediengattungen präsent: sowohl in Boulevardmedien und dem privaten Rundfunk als auch in der Qualitätspresse und dem öffentlich-rechtlichen Rundfunk. Innerhalb dieses Spektrums wirkten unsere Interviewpartner an einer ganzen Reihe unterschiedlicher Medienproduktionen mit, darunter TV-Magazine, politische Talkshows, TV-Reportagen und Dokumentationen. Außerdem sprachen die Befragten mit Journalist*innen für Radiosendungen, lokale und überregionale Boulevard- und Qualitätszeitungen und -zeitschriften sowie Print-Nachrichtenmagazine. Die meisten der Befragten hatten zahlreiche Kontakte zu verschiedenen Journalist $*$ innen, viele von ihnen waren jahrelang immer wieder in den Medien präsent. Einzelne Befragte hatten allerdings auch nur ein- oder zweimaligen Journalistenkontakt.

Vor diesem Hintergrund war zu erwarten, dass die Befragten unterschiedliche Erfahrungen im Umgang mit unterschiedlichen Journalist*innen gemacht hatten. Drei der Befragten waren Frauen, 15 der Befragten waren Männer, wobei keine geschlechtsspezifischen Unterschiede der jeweiligen Medienerfahrungen festgestellt werden konnten. Das Alter der Betroffenen variierte zwischen etwa 30 und 70 Jahren, wobei das durchschnittliche Alter bei etwa 45 Jahren lag. Die Interviews dauerten zwischen etwa $40 \mathrm{Mi}-$ nuten und drei Stunden mit einer durchschnittlichen Dauer von ca. 60 Minuten. Wir bemühten uns so lange um weitere Gesprächspartner*innen, bis das Interviewmaterial auf eine theoretische Sättigung schließen ließ (Fusch \& Ness, 2015). Hinweise auf eine theoretische Sättigung sahen wir darin, dass ab einem bestimmten Zeitpunkt der Datenerhebung durch zusätzliche Interviews keine grundlegend neuen Erkenntnisse mit Blick auf die uns interessierenden Aspekte von Medienerfahrungen mehr gewonnen werden konnten. Etwa ab dem zwölften Interview zeigte sich, dass sich die grundlegenden Muster der Informationen über den Kontakt mit unterschiedlichen Journalist*innen, Medienverarbeitung und Medienwirkungen im Kern wiederholten - was auf eine theoretische Sättigung mit Blick auf unsere zentralen Variablen schließen lässt.

Für den qualitativen Zugang sprach zum einen der defizitäre Forschungsstand, so dass es den Untersuchungsgegenstand explorativ zu ergründen galt (Lamnek 2005). Zum 
anderen erforderte die sensible Thematik des sexuellen Missbrauchs eine besondere Feinfühligkeit im Umgang mit den Befragten, die gerade im persönlichen Gespräch eher gewährleistet werden kann. Vor diesem Hintergrund haben wir uns durch verschiedene Maßnahmen zusätzlich bemüht, ein Vertrauensverhältnis zu unseren Befragten aufzubauen und sicherzustellen, dass die Teilnahme an unserer Studie für unsere Befragten mit - soweit bei der sensiblen Thematik möglich - keinen psychischen Zusatzbelastungen verbunden war. Auf folgende Aspekte haben wir deshalb besonders geachtet:

1. Wir haben bereits im Anschreiben unser kommunikationswissenschaftliches Forschungsinteresse möglichst ausführlich und transparent dargestellt, um dem Eindruck entgegenzuwirken, wir würden uns für potenziell re-traumatisierende Aspekte von Missbrauchserfahrungen (wie z. B. Tatdetails) interessieren. Auch haben wir bereits in der ersten Anfrage deutlich gemacht, dass alle Interviews anonymisiert ausgewertet würden.

2. Um zu verhindern, dass sich die angefragten Betroffenen unter Druck gesetzt fühlten, an unserer Studie teilzunehmen, haben wir im Falle einer Nichtbeantwortung unserer Anfrage auf eine systematische „Nachfassaktion“ verzichtet.

3. In jenen Fällen, in denen Befragte vor einer Interviewzusage weitere Informationen zu unserer Studie erfragten, haben wir Vorgespräche angeboten, in denen wir nochmals betont haben, dass wir keine Tatdetails abfragen und alle Aussagen anonymisieren würden. Auch haben wir darauf hingewiesen, dass selbstverständlich nicht alle Fragen beantwortet werden müssten. Nachdem die Betroffenen sich ausdrücklich zu einem Gespräch bereiterklärt hatten, wurde während oder im Anschluss an die Vorgespräche schließlich ein Termin für das eigentliche Interview festgelegt. In einigen Fällen erfolgten im Vorfeld der Interviews anstelle von Vorgesprächen auch mehrere Schriftwechsel, in denen wir den Befragten die o. g. Informationen gaben, diese uns aber auch bereits auf für sie relevante Medienberichte über ihren Fall hinwiesen. Insgesamt wurde bereits im Rahmen dieses Austauschs im Vorfeld der Interviews deutlich, dass der mediale Umgang mit dem Thema „sexueller Missbrauch “ für viele der angefragten Personen ein wichtiges Thema darstellte, über das sie bereits vor unserer Anfrage reflektiert hatten und zu dessen Erforschung die Befragten aktiv beitragen wollten.

4. Wenn ein persönliches Interview vereinbart wurde, sind wir bei der Wahl des Interview-Ortes den Wünschen der Befragten gefolgt, um es diesen zu ermöglichen, in einer vertrauten Umgebung über ihre Erfahrungen zu sprechen. So fanden beispielsweise Gespräche bei Befragten zuhause statt.

5. Bei der Interviewführung haben wir versucht, uns so gut wie möglich an unsere Gesprächspartner anzupassen, beispielsweise, was das Tempo des Gesprächs betraf. Für einige Interviewpartner war es leichter, ihre Medienerfahrungen abzurufen als für andere - entsprechend haben wir viele Gesprächspausen zugelassen, um es den Befragten zu ermöglichen, in Ruhe ihre Medienerfahrungen zu reflektieren. Auch haben wir darauf verwiesen, dass Fragen nicht beantwortet werden müssen, und darauf geachtet, bei kurzen Antworten auf einzelne Fragen keine mehrmaligen Nachfragen zu stellen, um zu verhindern, dass die Befragten sich unter Druck gesetzt fühlten, über Aspekte zu sprechen, die ihnen möglicherweise unangenehm waren.

6. In jenen Fällen, in denen die Befragten über (Medien)-Erfahrungen sprachen, die sie hätten identifizierbar machen können, haben wir den Befragten versichert, ihnen ihre Zitate vor einer Publikation vorzulegen. Im Rahmen dieses Austauschs im Nachgang der Interviews haben wir mehrere positive Rückmeldungen zu den Gesprächen bekommen, was uns für die darauffolgenden Interviews in dem beschriebenen Vorge- 
hen bestärkt hat und auch dazu führte, dass unsere Interviewpartner uns dabei halfen, weitere Gesprächspartner zu gewinnen.

Der Interview-Leitfaden (Hopf 2008) orientierte sich an den Modellkomponenten des oben beschriebenen Modells reziproker Effekte auf Betroffene sexuellen Missbrauchs. Er war damit in die folgenden Bereiche gegliedert: 1. Kontakt zu Journalist*innen (v. a. Motivation und Wege des Kontakts, Wahrnehmung der Interaktion), 2. Medienverarbeitung (v. a. Darstellungsbewertung, Medienwirkungsannahmen), 3. Medienwirkungen (Kognitionen, Emotionen, Verhalten).

Die Auswertung des Interviewmaterials erfolgte mithilfe einer qualitativen Inhaltsanalyse in Form einer inhaltlichen Strukturierung nach Mayring (2015). Ziel der Strukturierung ist es, „Material zu bestimmten Themen, zu bestimmten Inhaltsbereichen [zu] extrahieren und zusammen[zu]fassen“ (ebd.: 99). Für die vorliegende Untersuchung erschien diese Analysestrategie vor allem deshalb sinnvoll, weil die Medienerfahrungen unserer Befragten mit Blick auf verschiedene, im Vorfeld der Untersuchung deduktiv hergeleitete Themenbereiche untersucht werden sollten (siehe Kap. 3). Diese Themenbereiche wurden für die Analyse des Materials in folgende Haupt- und Subkategorien überführt:

Hauptkategorie 1: Kontakt zu Journalist*innen: Mit Blick auf die Erfahrungen im Kontakt mit Journalist*innen stellte sich auf Basis unserer theoriegeleiteten Vorüberlegungen zum einen die Frage, aus welchen Motiven heraus Betroffene sexuellen Missbrauchs den Kontakt zu Journalist*innen suchen und über welche Wege sie diesen herstellen. Zum anderen war für uns von Interesse, wie Betroffene die Interaktionen mit Journalist*innen wahrnehmen und welches journalistische Verhalten sie als positiv bzw. negativ bewerten. Entsprechend legten wir die folgenden Subkategorien für Hauptkategorie 1 fest: 1 . Motive für den Kontakt mit Journalist*innen, 2. Wege des Kontakts mit Journalist*innen, 3. Wahrnehmung der Interaktionen mit Journalist*innen.

Hauptkategorie 2: Medienverarbeitung: Vor dem Hintergrund unseres theoretischen Modells interessierte uns im Bereich der Medienverarbeitung zum einen die Frage, wie Betroffene sexuellen Missbrauchs unterschiedliche Medienberichte über ihren Fall bewerten und von welchen Wirkungsannahmen sie ausgehen. Außerdem war für uns von Interesse, welche Verhaltensänderungen sie nach Medienberichten über ihren Fall in ihrem sozialen Umfeld wahrnehmen und inwiefern diese ursächlich auf Medienberichte zurückgeführt werden. Entsprechend legten wir die folgenden Subkategorien für Hauptkategorie 2 fest: 1 . Bewertung der Berichterstattung, 2. Wirkungsannahmen, 3. Wahrnehmung von Verhaltensänderungen.

Hauptkategorie 3: Medienwirkungen: Im Bereich der Medienwirkungen interessierten wir uns sowohl für emotionale als auch für kognitive und behaviorale Medienwirkungen auf Betroffene sexuellen Missbrauchs. Wir legten entsprechend folgende Subkategorien für die Hauptkategorie 3 fest: 1. Wirkungen auf Emotionen, 2. Wirkungen auf Kognitionen, 3. Wirkungen auf Verhalten.

Alle Aussagen, die sich mit den Medienerfahrungen der Betroffenen beschäftigten, wurden einer oder mehreren der beschriebenen Kategorien zugeordnet und in einem mehrstufigen Verfahren immer weiter zusammengefasst, bis für jede Kategorie ein komprimierter Textkorpus entstand, der Aussagen auf einer abstrakteren Ebene erlaubte. Die Auswertung der Interviews erfolgte mithilfe der Analysesoftware MAXQDA.

Um die Anonymität der Interviewpartner zu gewährleisten, wird für alle Befragten im Folgenden die männliche Form verwendet, die Befragten werden als B1-18 bezeichnet. 


\section{Befunde}

\subsection{Kontakt zu Journalist*innen}

\subsubsection{Motive für den Gang in die Öffentlichkeit (Forschungsfrage 1.1)}

Zahlreiche Befragte befanden sich vor ihrem Schritt in die Öffentlichkeit in einer (Ausgangs-)Situation, in der sie Ohnmacht gegenüber Strafjustiz und Kirche empfanden: Weil die Betroffenen erst viele Jahre nach dem Missbrauch (der zumeist in der Kindheit stattfand) über das Erlebte sprechen konnten, ließen sich Täter aufgrund von Verjährungsfristen nicht mehr belangen. Gleichzeitig wurden die Aufarbeitungsbemühungen der Kirche als unzureichend wahrgenommen - was sie in vielen Fällen schließlich zum Gang in die Öffentlichkeit motivierte: „Die Presse war für mich wirklich die letzte Instanz, nachdem ich gesehen habe, dass alle, die zuständig gewesen wären oder die Verantwortung gehabt bätten, alle, die die Möglichkeit und Pflicht gehabt hätten, gegen meine Täter vorzugehen, das nicht tun" (B5). Der Gang in die Öffentlichkeit zielte also darauf ab, im Sinne von Selbstermächtigung Kontrolle zurückzuerlangen, potenzielle Opfer zu schützen und anderen, (noch) nicht sprechfähigen, weil traumatisierten Opfern „eine Stimme“ zu geben: „Ich will den Kampf kämpfen und ich kämpfe ihn auch für andere Betroffene. Ich muss kämpfen, so lange es irgendwie geht. Dass sich in der Gesellschaft was ändert, diese Scheinheiligkeit, dieses Nicht-Hinschauen, dass man es einfach nicht wabrbaben will, obwobl es eigentlich omnipräsent ist" (B1).

Es ging Befragten aber nicht nur darum, auf Missstände und die Sicht der Betroffenen aufmerksam zu machen, darüber hinaus wurde die Medienöffentlichkeit gesucht, um politische Forderungen an Politik und Kirche wirksam zu vertreten. So ging und geht es den Betroffenen darum, die Verjährung von sexuellem Missbrauch aufzuheben und kirchliche Reformen zu erzwingen, die an den strukturell-systemischen Ursachen von Missbrauch ansetzen. Betroffene sollten daher nicht „in ibrem stillen Kämmerlein leiden" (B3).

Neben diesen eher politischen Motiven wurden auch ganz persönliche Motive für den Schritt in die Öffentlichkeit genannt. So ging es manchen Betroffenen auch um die Anerkennung ihres persönlichen Leids und die persönliche Aufarbeitung des Erlebten, etwa um die Überwindung ihrer Schamgefühle. Besonders bemerkenswert erscheint in diesem Zusammenhang, dass zwei der Befragten auch davon berichteten, sie wollten durch ihren Gang in die Öffentlichkeit ihre Angst überwinden, selbst zum Täter zu werden. So sagte B1: „Es gibt auch die Angst, dass ich vom Opfer zum Täter werde. Und ich habe gesagt, durch dieses Publizieren möchte ich diese Schiene durchbrechen". Gleichwohl ist zu betonen, dass die meisten Befragten deutlich machten, sie hätten ihre Missbrauchserfahrungen bereits vor ihrem ersten Kontakt zu Journalist*innen relativ gut aufgearbeitet.

Bemerkenswert erscheint, dass laut B6 in manchen Fällen auch Kirchenvertreter, die anonym bleiben wollten, Betroffene sexuellen Missbrauchs zur Kontaktaufnahme mit Journalist*innen ermutigten. Im Rahmen solcher Instrumentalisierungsversuche hieße es dann: "Ja, bleibt weiterhin in der Öffentlichkeit, das ist wichtig. Aber nein, ich will nicht genannt werden, keinesfalls".

Schließlich sprach ein Befragter auch von einer gewissen Sucht nach Medienaufmerksamkeit für den eigenen Fall: „Das macht auch die Berichterstattung so beikel, dass es zum größten Teil Menschen sind, die verwundet geblieben sind. Die an dem, was sie erlebt haben, ibr Leben lang knabbern. Denen auch etwas weggenommen worden ist. Zum Beispiel dieses Gefübl, geliebt zu werden. Aufmerksamkeit beißt ja nur: Du bist wichtig, du erfährst Wertschätzung. Es gibt Menschen, die dich kennen und die sich für 
dich interessieren. Ich glaube, wenn man das im Leben nicht hat - und viele Betroffene haben das einfach nicht wegen der Tat - können Medienberichte diese Seite einnehmen" (B5).

\subsubsection{Wege des Kontakts (Forschungsfrage 1.2)}

Die Befragten suchten den Kontakt zu Journalist*innen in der Regel gezielt, etwa über persönliche Mails oder anonyme Online-Briefkästen. Letzteres war zum Beispiel für B5 hilfreich: „Ich war sebr abnungslos und babe nicht gewusst, auf welchen Wegen ein normaler Mensch, der noch keinen Kontakt mit Medien hatte, einen solchen Kontakt aufnimmt. Ich habe dann auf [Name einer Online-Nachrichtenseite] gesehen, dass es einen anonymen Briefkasten gibt. Da habe ich einfach mal hingeschrieben, um zu gucken, ob da eine Reaktion käme und ob da jemand Interesse hätte - und das war dann auch so." Auch über öffentliche Veranstaltungen zum Thema Missbrauch und über journalistische Anfragen bei Opfervereinen gelangten Betroffene in Kontakt mit Journalist*innen.

Gerade jene Befragte, die eigeninitiativ Öffentlichkeit suchten, wählten die Medien hierfür sorgsam aus. Es sei wichtig, mit „seriösen“ Medien zu arbeiten und Boulevardmedien angesichts der Gefahr eines Kontrollverlusts über die eigene Darstellung abzulehnen. B1 erzählte, er habe gezielt an einem Beitrag für ein Fernsehmagazin mitgewirkt, das in seinen Augen seriös sei und gleichzeitig auch die „heißen Eisen“ anfasse. Es ging dem Befragten also um eine drastische Darstellung seines Falls, ohne gleichzeitig (erneut) einen Kontrollverlust zu erleben. Interessant erscheint, dass beispielsweise B3 betont, er würde alle Anfragen von Regionalmedien ablehnen, da er „hier in der Gegend nicht öffentlich als Opfer auftreten wolle“, andernfalls käme es zu Reaktionen des direkten sozialen Umfeldes im Sinne einer „sekundären Viktimisierung“.

Neben solchen generellen Überlegungen zur Medienauswahl machten die Befragten ihren Medienkontakt offenbar auch von einzelnen Journalist*innen abhängig. Wer als vertrauenswürdig gelten kann und wer nicht, über solche Fragen könnten sich Betroffene laut B5 auch im Internet austauschen: „Es spricht sich herum, welche Journalisten vertranenswürdig sind und welche weniger. Oder was man beachten soll, wenn man an die Öffentlichkeit gebt.“

\subsubsection{Wabrnebmung der Interaktion mit Journalist*innen (Forschungsfrage 1.3)}

Mehrere Befragte berichteten zunächst von überwiegend positiven Erfahrungen in der Interaktion mit Journalist־innen. Die Medien hätten im Großen und Ganzen gute Arbeit geleistet, gerade Vertreter öffentlich-rechtlicher Medien wurden als professionell und empathisch beschrieben. Die Befragten würdigten gerade Journalist*innen, die ihnen das Gefühl maximaler Kontrolle über die Interviewsituation vermittelt hätten, indem sie ihnen zum Beispiel die Möglichkeit zum jederzeitigen Interviewabbruch und zur Autorisierung ihrer Aussagen einräumten. Erleichtert worden sei die Interviewsituation auch durch persönliche Vorgespräche und eine ausführliche Hintergrundrecherche der Journalist\%innen.

Gleichwohl kamen die Befragten im weiteren Gesprächsverlauf intensiver auf zahlreiche Interaktionen mit Journalist*innen zu sprechen, die sie als unangenehm wahrgenommen hatten, nicht zuletzt aufgrund ihres Potenzials zur Re-Traumatisierung. Gerade die Forderung, den erlebten Missbrauch ausführlich und mehrmals zu schildern, kann für Betroffene offenbar sehr belastend sein. So berichtete beispielsweise B10, er sei von einem Journalisten zu Details seines Missbrauchs „fünf Mal das Gleiche gefragt [worden], bis ich es so gesagt habe, wie sie sich das vorstellen". Auch B18 berichtete von 
unangenehmen Interviewsituationen, in denen ihn Journalist*innen durch Fragen zu Tatdetails gezielt zum Weinen bringen wollten, was er als „schäbig und unethisch“ empfunden habe. Ähnlich wie B18 sahen sich auch weitere Befragte zur Publikumsunterhaltung instrumentalisiert. B5 erzählt: „Was mich dann sebr gestört hat [...], wenn sebr in diese Richtung gefragt wird, in Details, in dieses Sexuelle hinein ". Dieses journalistische Interesse an intimen Details ging in einigen Fällen so weit, dass sich Befragte gezwungen sahen, vor allem Journalist*innen von Privatsendern deutlich in die Schranken zu weisen: „Einem habe ich wirklich mal gesagt: ,Ich werde Ibnen jetzt nicht erzäblen, was mir wann wo wie in welche Körperöffnungen reingesteckt worden ist, weil ich das nicht erzäblen will“" (B12).

Um an derartige Infos zu gelangen, schreckten manche Journalist*innen auch vor Manipulationsversuchen nicht zurück. So berichtet B12 davon, eine Journalistin hätte ihm nach einem Interview, allerdings vor noch laufender Kamera, unvermittelt eröffnet, sie sei ebenfalls sexuell missbraucht worden und würde gerne wissen, wie sie nun damit umgehen solle. „Bei dieser Journalistin hatte ich das Gefübl, die wollte da irgendwas bei mir rausdrücken, das war nicht ebrlich", so der Betroffene. Nach Wahrnehmung von Betroffenen geht es manchen Journalist*innen also auch darum, persönlichen „Voyeurismus" (B6) zu befriedigen. B6 machte dies am Beispiel einer Fernsehredakteurin deutlich, die im Rahmen der Vorbereitung einer Sendung wie folgt auf ihn zukam: „Dann meinte sie, dass das jetzt nicht in der Sendung gefragt wird, aber nur mal für sie persönlich würde sie gerne wissen, ob er mich wirklich penetriert hat [...] das habe ich schon immer mal wieder erlebt. Selten so unverboblen wie da, [...], aber in Abstufungen auch immer mal wieder von anderen Journalisten".

Vor dem Hintergrund der Gefahr einer Re-Traumatisierung ist auch die negative Wahrnehmung von Situationen zu sehen, in denen Journalist*innen wollen, dass Betroffene für Filmaufnahmen an die Tatorte zurückkehren. Laut B12 würde dies aus journalistischer Perspektive zwar „einen guten Eindruck im Beitrag“ machen, Betroffene könnten dies jedoch als psychisch belastend empfinden. Ähnlich negativ wurde das Verhalten von Journalist*innen gerade auch dann wahrgenommen, wenn sie „so einen Tunnelblick bekommen und sagen: ,Ich brauche jetzt aber [...] noch einen Abgang, ich brauche noch einen Aufgang, ich brauche noch die Einstellung und ich will die Frage unbedingt beantworten", dann verlieren sie ibre Empathie" (B12).

Als unangenehm wurden darüber hinaus Situationen wahrgenommen, in denen sich Betroffene von Journalist*innen unter Druck gesetzt sahen und dadurch einen Kontrollverlust fühlten. Problematisch sei, „wenn man den Eindruck hat, die Geschichte ist schon fertig und jemand braucht nur noch Text, den er in Lücken füllen kann, um es entsprechend auszuschmücken. Also alles, was auf dieser Ebene ist, wo dieser Respekt fehlt, Druck gemacht wird, und wo man das Gefübl bekommt, es setzt sich jemand über einen binweg“ (B5). Die Befragten nahmen also in verschiedenen Situationen Journalist*innen als Akteure wahr, die für ihre Geschichte noch „irgendein Opfer von Gewalt in der Kirche " gebraucht hätten, das in ihre Geschichte noch „schön reinpassen“ würde (B5). Auch bemängelten einige Befragte, dass Journalist*innen nur dann mit ihnen kommunizieren würden, wenn sie gerade für einen Medienbeitrag gebraucht würden. Nach Wahrnehmung von Befragten ließen Journalist*innen damit persönliches Interesse und Respekt ihnen gegenüber vermissen.

In diesem Zusammenhang klagten Befragte deutlich über eine in vielen Fällen unzureichende Interview-Vorbereitung der Journalist*innen, mitunter seien auch nur Praktikanten geschickt worden. Die ohnehin als „brutal stressig“ (B3) empfundenen Interaktionen mit Journalist*innen würden noch anstrengender, wenn Betroffene zusätzlich 
Hintergrundinformationen liefern und deshalb ihr "geistiges Skript“ (B8) verlassen müssten. Dies könne unter Umständen auch zu detaillierten Erklärungen bestimmter Sachverhalte führen, die Betroffene „eigentlich nicht veröffentlicht haben wollten“ (B8).

Einzelne Befragte betonten schließlich auch, sie hätten bestimmte Verhaltensweisen von Journalist $*$ innen mitunter als sehr verletzend und respektlos wahrgenommen. Dafür einige Beispiele: B5 berichtete, er habe es als sehr respektlos empfunden, dass zwei unterschiedliche TV-Redaktionen durch das Anbieten immer höherer Gagen um ihn „gefeilscht" hätten. B12 beklagte, dass eine Fernsehanfrage an ihn wieder zurückgezogen worden sei, weil man nun eine „Professorin“ für die Show gewonnen habe. Einen besonders drastischen Fall einer journalistischen Grenzüberschreitung beschreibt B17, der nach Bekanntwerden seines Missbrauchs von einer „Meute“ Journalist*innen vor seiner Wohnung „von morgens bis abends belagert" worden sei. Dabei sei er diverse Male von Vertretern der „Klatschpresse“ gegen seinen Willen fotografiert worden, die Journalist*innen seien gar „noch aufdringlicher“ geworden, als er sie gebeten habe, ihn in Ruhe zu lassen. Er berichtete, einige Medienvertreter hätten versucht, sich Zutritt zu seiner Wohnung zu verschaffen, indem sie vorgaben, ihm Zeitungs-Abonnements verkaufen $\mathrm{zu}$ wollen. Er habe sich schließlich gezwungen gesehen, die Polizei einzuschalten und den Namen auf seinem Klingelschild zu ändern. Diese Erfahrung sei für ihn ein „Martyrium “ gewesen, das Selbstmordgedanken ausgelöst habe, weil „die einen einfach nicht zufriedengelassen haben".

\subsection{Medienverarbeitung (Forschungsfrage 2)}

\subsubsection{Bewertung der Berichterstattung}

Die meisten Befragten zeigten sich überwiegend zufrieden mit der Berichterstattung über ihren Fall, trotzdem kritisierten sie verschiedene Aspekte der Berichterstattung mitunter scharf. Möglicherweise erklärt sich diese Diskrepanz dadurch, dass die Befragten es vor dem Hintergrund ihrer schwierigen Ausgangssituation (vgl. Kap. 5.1.1) als überaus positiv bewerten, wenn Medien überhaupt das Thema Missbrauch in der Kirche aufgreifen und Betroffenen so das lang ersehnte öffentliche Gehör schenken. In diesem Zusammenhang wurde kritisiert, dass sexuelle Gewalt in der Kirche vor der Berichterstattungswelle im Jahr 2010 kaum medial thematisiert worden sei und bis heute nur anlassbezogen und damit in Wellen auf der Medienagenda stehe.

Darüber hinaus zeigten die Befragten sehr differenzierte Bewertungen der Berichterstattung in öffentlich-rechtlichen und privaten Medien. Obwohl auch negative Erfahrungen mit öffentlich-rechtlichen Medien berichtet wurden, erhielt deren Berichterstattung generell eine positivere Bewertung: „Die Privaten dramatisieren, das ist einfach so. Und beim WDR, auch beim ZDF, hatte ich das Gefühl, da kommt das tatsächlich rüber, was sie gedrebt haben, während ich bei Sat.1 und RTL zweimal das Gefühl hatte, die haben es dramatischer gemacht, als es sein musste" (B12). Auch B10 lobte die Journalist*innen des öffentlich-rechtlichen Fernsehens dafür, dass sie „nicht sensationsgeil“ über ihn berichtet und „nichts verdreht“ hätten.

Fokussieren Medienberichte hingegen zu sehr auf die persönliche Geschichte der Betroffenen und Tatdetails, so sehen die Befragten ihre - mitunter dezidiert politischen - Botschaften durch Emotionalisierung und „Sensationslust“ zu sehr in den Hintergrund gerückt. „Wenn man mich als Opfer darstellen möchte, um irgendwie Gefüble zu wecken, um zu emotionalisieren mit der Geschichte. Das ist für mich schon das Schlimmste, was ich bei Journalisten erlebt habe" (B5). Auch B6 berichtete davon, er hätte bei manchen Zeitschriftenberichten das Gefühl gehabt, seine Geschichte würde genutzt, um „die äl- 
teren Damen beim Frisör zu unterhalten“. B5 führte hierzu aus: „Es gibt gerade in deutschen Medien dieses Gefübl, dass das Publikum oder die Leserschaft ein bisschen dumm ist, dass man ibnen das erklären und das vereinfachen muss, weil sie es sonst nicht versteben. Das ist [...] fatal, weil man dann ganz schnell in diesen Voyeurismus und diese Leidenspornographie kippt und damit weder dem Publikum noch den Betroffenen gerecht wird, noch einen Aufklärungseffekt erzielt, sondern ganz schlechte, abgeschmackte Unterhaltung abliefert".

Gleichwohl wünschten sich die Befragten drastische Darstellungen der Thematik, im Idealfall sollten Berichte sachlich und faktenorientiert, aber dennoch emotional sein. B1 bemerkte zu diesem Spagat: „Es gibt da natürlich einen Zwiespalt zwischen VoyeurJournalismus und Blümchen-Stil. Aber wenn man das nur im Blümchen-Stil beschreibt, dann wacht auch keiner davon auf. Ich will schon auch in aller Klarbeit diese Fratze und die Hässlichkeit zeigen, die da ist. Das ist nicht nur ein sanftes Streicheln. Das hat mich psychisch krank gemacht".

Ein zentrales Defizit sahen zahlreiche Befragte in einem Mangel an Hintergrundberichterstattung; geäußert wurde der Wunsch nach einem stärkeren medialen Fokus auf die gesellschaftlichen und kirchenstrukturellen Ursachen von sexuellem Missbrauch. B5 sah hier immerhin eine positive Entwicklung: „Man merkt zumindest in den letzten ein bis zwei Jahren, dass Medien das schon begriffen haben und immer mehr von Machtmissbrauch die Rede ist. Dass die Strukturen mehr in den Blick genommen werden. Das ist ganz wichtig und auch ein Erfolg. Obne Medien wäre ganz viel nicht passiert, was passiert ist. Die haben auch ganz viel richtiggemacht“. Allerdings wollen manche Betroffenen nicht, dass Journalist*innen dabei ihre persönlichen Missbrauchserfahrungen für pauschale Kritik an Kirchenstrukturen instrumentalisieren. Es passiere ständig und sei ärgerlich, so ein Betroffener, „wenn meine Geschichte immer als Kritik an der Kirche oder Gegnerschaft zur Kirche erzählt wird. Wenn diese Gegenüberstellung erfolgt. Auf der einen Seite die Kirche und auf der anderen Seite ich. Dass nicht respektiert und gar nicht verstanden wird, dass ich in der Kirche und Teil der Kirche bin. Und ich nicht gegen die Kirche gehe, indem ich meine Geschichte erzäble"(B5). Auch die Frage, ob Betroffene als „Opfer“ bezeichnet werden möchten, variiert offenbar von Fall zu Fall. Während manche der Befragten diese Bezeichnung als legitim empfinden, möchte beispielsweise B1 dieses „Etikett“ in Medienberichten vermeiden. Er begründet: „Dann mache ich mich nochmal klein und ich möchte nicht mehr klein sein". Journalist*innen könnten hier also auf die individuellen Wünsche ihrer Interviewpartner eingehen.

Kritisch sahen mehrere Befragte gerade Lokalmedien, deren Berichterstattung als „parteiisch“ und kirchennah bezeichnet wurde. Laut B4 würde eine Lokalzeitung in seiner Region zwar manchmal auch kritisch über Missbrauchsfälle berichten, allerdings würde dann unmittelbar zuvor oder danach ein kirchennaher Bericht folgen: „Der gleiche Reporter, der den Schlag in die Magengrube verteilt, verteilt vorber oder nachber ein Pflaster". Im Einklang damit betonte B7: „Manchmal habe ich das Gefübl, dass es ein Text ist, den der Sekretär vom Bischof geschrieben haben könnte“. B3 führte dies auf die in vielen Regionen starke Verankerung der Kirche im wirtschaftlichen und gesellschaftlichen Leben zurück. So habe eine Lokalzeitung ihn wie folgt gezielt diskreditiert: „Die haben mich im Prinzip als irre dargestellt, also mehr oder weniger die Version von der Diözese übernommen. [...] also das war für mich sehr, sehr schlimm, also diese Diskreditierung". Vor diesem Hintergrund fordert zum Beispiel B3, nur dann über sexuellen Missbrauch zu berichten, wenn man sich als Journalist auch dazu entschließt, dem jeweiligen Betroffenen zu glauben und seine Schilderungen nicht öffentlich anzuzweifeln. 


\subsubsection{Wirkungsannabmen}

Die bereits erwähnten Versuche von Betroffenen, über Medienberichte öffentlichen Druck auf politische und kirchliche Entscheidungsträger aufzubauen, legen entsprechende Medienwirkungsannahmen nahe. Grundsätzlich wurden Medienwirkungen auf verschiedene Teilpublika angenommen. Hierzu gehörten zunächst andere Betroffene sexuellen Missbrauchs, die, so die Annahme von B10, durch Medienberichte und die damit verbundene Enttabuisierung der Thematik ermuntert würden, auch über ihren eigenen Missbrauch zu sprechen. „Da gebt es nicht unbedingt um die große Öffentlichkeit, sondern wenn vielleicht jemand in meinem Umkreis frei und offen drüber reden kann, dann öffnet sich die Person auch leichter, wenn sie merkt: Ja, ich muss mich nicht dafür schämen"“.

Kritisch sah B8 die auf Betroffenen-Seite angeblich vorherrschende „Fantasie“, eine möglichst emotionale Fallschilderung zöge auf kirchlicher Seite einschneidende Konsequenzen nach sich. Infolge dieser Annahme würden sich Betroffene in den Medien in einer Detailliertheit und Drastik zu ihrem Fall äußern, die wiederum dazu führe, dass sie auch im sozialen Umfeld immer wieder mit ihrem „Opferstatus“ konfrontiert würden - während die erhofften Zugeständnisse der Kirche ausblieben. B8 vertrat die Ansicht, besonnene, sachliche Äußerungen Betroffener könnten in den Medien politisch viel mehr bewegen als emotionale Schilderungen, man bliebe so ein ernstzunehmender Verhandlungspartner mit Einfluss. "Jeder Medienbericht half, die Aufmerksamkeit auf uns zu baben und weiter Gewicht zu haben und das Gewicht heißt ja auch: Ich habe sozusagen Macht, ich habe Einflusspotenzial und bin damit auch ein gewichtiger Verhandlungspartner".

Bemerkenswert erscheint (vor dem Hintergrund eines mitunter als "voyeuristiscb“ wahrgenommenen journalistischen Verhaltens) die auf Betroffenen-Seite geäußerte Vermutung, Rezipienten der Berichterstattung würden diese zur Unterhaltung verfolgen. So machte B8 deutlich, dass er bei emotionalisierenden Aspekten der Berichterstattung denke, diese gäben dem Publikum einen „Kick“, während sich das Publikum ohne Emotionalisierung in der Berichterstattung langweilen würde. B1 äußerte gar die Angst, seine Geschichte könne durch eine mediale Thematisierung zu einem weiteren „Missbrauch“ führen: „Da kam mir dann irgendwann der Gedanke [..]: Wenn der Täter jetzt den Bericht sieht... Ich weißja, wie diese Pädokriminellen ticken, ich weiß, dass sie damit angeben, dass sie jemanden beherrschen, dass sie mit ihm alles machen können und so weiter. Und diese Vorstellung, dass dann sich jemand im Nachbinein nochmal dran aufgeilen könnte, das find ich schlimm".

Auch weitere Befragte berichteten von ihrer Angst vor unerwünschten Wirkungen der Medienberichte über ihren Fall. B5 „hatte Angst, dass meine Täter irgendwie gegen mich vorgehen. Ich hatte Angst [...], dass es falsch dargestellt wird [...]. Ich hatte Angst, dass Menschen, die das lesen, mich irgendwie angreifen oder meinen, ich wäre selbst schuld. Oder dass eine Form von victim shaming stattfindet". Auch die Aussagen weiterer Befragter weisen darauf hin, dass die Angst vor sozialer Ausgrenzung bei vielen Betroffenen im Vorfeld von Berichterstattung allgegenwärtig war.

\subsubsection{Verbaltensänderungen}

Mit Blick auf Reaktionen der Kirche betonten mehrere Befragte, ohne die Hilfe der Medien wären sie von der Kirche überhaupt nicht als Opfer wahrgenommen worden und hätten keine Entschädigung bekommen. B1 resümierte: „Ich habe nicht viel erreicht, aber obne die Presse bätte ich gar nichts erreicht. Obne die Presse bätte die Kirche sich 
gar nicht bewegt“. Allerdings erhielten mehrere Befragte nach Medienberichten über ihren Fall auch unangenehme Reaktionen, darunter Vorwürfe und juristische Drohungen. So berichtete beispielsweise B5, seine Täter hätten gegen emotional zugespitzte Medienberichte geklagt, was ihm "handfeste Schwierigkeiten“ eingebracht hätte. Er erzählte: „Das ist nur zurückzufübren auf Journalisten, die so zugespitzt haben, dass sie meinen Tätern eine Angriffsfläche geboten haben. Was ich selbst immer versuche zu vermeiden, wenn ich irgendwo spreche“.

Das soziale Umfeld aus Freunden, Bekannten und der Internetgemeinschaft reagierte nach Wahrnehmung der Befragten überwiegend positiv, wenn auch mitunter überrascht auf die Berichterstattung. B5 sagte vor diesem Hintergrund: „Ich habe das Gefühl, die Öffentlichkeit ist der Richter, den ich mir die ganze Zeit gewünscht habe. Die Instanz, die sagt, das hätte nicht passieren dürfen und die mir das auch zuspricht. Ich erlebe viele Menschen, die etwas über mich gelesen, gehört oder gesehen haben und mich kontaktieren, um mir genau das zu sagen. Die mir - gar nicht so sebr ibr Mitgefübl-eher ibre Bewunderung, Zustimmung, Solidarität zum Ausdruck bringen. Das tut mir sebr gut“. Auch wurde betont, andere Betroffene hätten durch die Medienbeiträge den Mut gefasst, „den Mund aufzumachen" und Anzeige zu erstatten. Bemerkenswert erscheint, dass B6 betonte, er hätte bestärkende Publikumsreaktionen nur nach Berichterstattung in informationsorientierten Medien erhalten, nicht nach Veröffentlichungen in unterhaltungsorientierten Publikumszeitschriften.

Allerdings berichteten Befragte auch von vereinzelten negativen Reaktionen. Sie sahen sich neben Beschimpfungen in der Online- und Offline-Welt auch Vorwürfen aus den Reihen der Kirchengemeinde ausgesetzt: „Die haben mich da zwei Tage malträtiert: , Sie werden dann zum Täter und der arme Mann wird zum Opfer, der kann doch so gut mit Kindern, der macht Videoabende und Pizzaabende und der verliert doch jetzt seinen Lebenssinn" (B1). Auch von negativen Reaktionen im Familien- und Bekanntenkreis berichteten verschiedene Befragte, darunter B1: „Meine Mutter hat gesagt: ,Jetzt hast du 40 Jahre geschwiegen, hättest du nicht noch 40 Jabre die Klappe halten können?" Und mein Vater hat gesagt: , Wegen dir können wir uns jetzt im Dorf nicht mehr blicken lassen“". B3 berichtete, ein Nachbar habe zu ihm nach einer Fernsehsendung gesagt: „Statt dich ins Fernseben zu setzen, würdest du besser daran tun, wenn du mal wieder in die Kirche geben würdest“. Drastisch schilderte auch B16 negative Erfahrungen nach Medienberichten über seinen Fall. So sei er von Personen in seinem sozialen Umfeld folgendermaßen auf Medienbeiträge angesprochen worden: „Ich habe dich im Fernseben geseben, $d u$ bist doch der [...], der mit seinem Lebrer gevögelt hat."

\subsection{Wirkungen (Forschungsfrage 3)}

\subsubsection{Emotionen}

Nur wenige Befragte bescheinigten der (Medien-)Öffentlichkeit einen Beitrag dazu, ihre Missbrauchserfahrungen zu verarbeiten. Mehrheitlich wurde die Ansicht vertreten, mediale Aufmerksamkeit sei mit großem Stress verbunden. Einige Betroffene, gerade Personen, die sich vor einer abgeschlossenen Therapie an die Medien wenden, würden infolge von Berichterstattungswellen irgendwann „Zusammenklappen“ (B3). Berichtet wurde beispielsweise von Kreislaufzusammenbrüchen, dissoziativen Zuständen, Lähmungserscheinungen und Suizidversuchen.

Die ständige Konfrontation mit den eigenen Missbrauchserfahrungen und Gefühle von Scham wurden als belastend empfunden. B5 erinnerte sich: „Das war schon überwältigend [...], als die ersten Male mein Gesicht, mein Bild, mit dabei war. Ich habe mich 
da immer noch vor allem geschämt. Ganz große Scham darüber, dass Menschen, die mich kennen, auch meine Geschichte kennen und dass ich das nicht mebr loswerde“. Weil schon relativ sachliche Berichte über den eigenen Missbrauch als sehr belastend empfunden wurden, verwundert nicht, dass bestimmte Berichte von Betroffenen als unerträglich empfunden wurden. Ein Bericht, der die Integrität eines Befragten in Frage stellte, habe ihn „richtig traumatisiert“. Auch wenn Betroffene das Gefühl haben, die Kontrolle über ihre mediale Darstellung zu verlieren, kann dies massive emotionale Folgen haben: „Das hat eine Trigger-Gefahr. Das habe ich auch erlebt. Nicht so sebr durch die Art der Frage oder weil Erinnerungen an die Tat wieder hochkommen [...], sondern weil dieses Gefühl, dass sich jemand über einen hinwegsetzt, triggert. Wie die Missbrauchserfabrung, wo sich auch jemand über einen hinweggesetzt hat. Dieses Gefübl, da macht jemand, was er im Kopf hat und was er will. Wie es mir dabei geht und was ich will, ist dabei unwichtig. Das war in solchen Momenten sehr belastend für mich" (B5).

Die Emotion Wut kann nach Ansicht von Befragten ausgelöst werden, wenn Medien die Reaktionen der Kirche auf Missbrauchsfälle unkritisch wiedergeben. Hingegen kann eine kritische Berichterstattung positive Emotionen hervorrufen, darunter „Genugtuung “ gegenüber den Tätern, verbunden mit dem Gefühl, weniger einsam und respektiert $\mathrm{zu}$ sein. Dem Interviewmaterial ist auch zu entnehmen, dass die zunächst wohltuende Medienaufmerksamkeit für emotional vulnerable Betroffene jedoch ein zweischneidiges Schwert sein kann. Da die Medien nur an wenigen Betroffenen dauerhaft Interesse zeigten, würde die erhoffte Aufmerksamkeit und Zuwendung dritter Personen (wenn überhaupt) nur punktuell erfolgen.

\subsubsection{Kognitionen}

Die Interviews zeigen, dass Berichte über die Betroffenen auch deren Realitätsvorstellungen und Einstellungen gegenüber Medien geprägt haben. Die wohl wichtigste Erkenntnis war für die meisten Befragten, dass die meisten Journalist*innen primär das breite Publikum im Blick haben und bei Berichten über Betroffene einem (aufmerksamkeits-)ökonomischen Primat folgen: „Ich entdecke da schon viel Sensationslust, ich unterstelle größtenteils doch als Triebfeder das Thema Auflagenzablen und Einschaltquoten" (B6). Neben der negativ bewerteten Wahrnehmung, Medien würden nur in Wellen über die Thematik berichten, hat die Berichterstattung bei Betroffenen das Bild gefestigt, „dass sich die Journalisten relativ wenig Gedanken machen, was das alles auslöst. Wie es dem Interviewpartner oder dem Opfer letzten Endes geht, steht auf alle Fälle nicht im Fokus des Journalisten“ (B10). Es sei „vielen Journalisten einfach nicht bewusst, dass es Täter gibt, die bereit sind, gegen ibre Opfer vorzugehen, dass sich die Opfer durch diese Interviews einer Gefahr aussetzen" (B5). Umgekehrt würden Journalist*innen sich selbst und ihre Medienhäuser sehr wohl vor Reaktionen der Kirche schützen - beispielsweise, indem sie in „heiklen“ Fällen komplett darauf verzichten würden, über Missbrauch zu berichten, oder aber Täter komplett anonymisierten. Der Einfluss der Kirche wurde mitunter als "gigantisch“ (B1) beschrieben, mehrere Befragte sahen enge Verknüpfungen zwischen Kirche und Medienhäusern.

\subsubsection{Verbalten}

Die Interviews legen nahe, dass die Verhaltensreaktionen von Betroffenen generell maßgeblich von ihrer Bewertung der Berichterstattung und den Reaktionen des sozialen Umfeldes abhängen. So mündete negativ wahrgenommene Berichterstattung in Versuchen, mit Journalist*innen Kontakt aufzunehmen, um entweder eine bestimmte, an- 
dere Berichterstattung herbeizuführen, oder aber auch um die Löschung bestimmter Online-Berichte zu erwirken. Letzteres erfolgte, um in der öffentlichen Wahrnehmung nicht auf die Rolle als Opfer sexuellen Missbrauchs reduziert zu werden: „Die haben das dann rausgenommen, die Links, weil ich gesagt habe: ,Jetzt reicht es, jetzt ist gut, jetzt möchte ich das nicht mehr" (B3). In einem anderen Fall sah sich ein Betroffener gezwungen, mit juristischen Schritten zu drohen, um eine Gegendarstellung eines diskreditierenden Medienberichts zu erwirken. Um die Wirkungen der seiner Ansicht nach „parteiischen“ Missbrauchs-Berichte einer Lokalzeitung abzumildern, bat B3 diese darum, Informationen für Hilfesuchende als Disclaimer zu veröffentlichen. Dies wurde in der Literatur bereits als ein journalistisches Qualitätskriterium für die Berichterstattung über SKM genannt (Döring \& Walter 2019).

Gleichwohl zogen sich einige Befragte nach negativen Erfahrungen mit den Medien auch zurück, oder sie gingen selektiver bei der Beantwortung von Medienanfragen vor. So entschied sich B6 nach negativen Erfahrungen in einer Talkshow dazu, Anfragen für ähnliche Formate kategorisch abzulehnen und kritischer zu prüfen, ob es Medien „nur um Unterhaltung" gehe, oder ob auch ein journalistisches Interesse an seinen politischen Botschaften bestehe. B15 berichtete, er habe infolge seiner Unzufriedenheit mit der fehlenden Hintergrundberichterstattung über sexuellen Missbrauch seine eigenen OnlineMedieninhalte produziert. So habe er ein Gegengewicht zu der in seinen Augen gewaltfokussierten, oberflächlichen Medienberichterstattung schaffen wollen.

Es finden sich gleichwohl auch Hinweise darauf, dass positiv bewertete Berichterstattung unterschiedliche Verhaltenswirkungen zur Folge haben kann. Mehrere Befragte berichteten, die Berichterstattungswelle im Jahr 2010 habe sie motiviert, über ihre Missbrauchserfahrungen zu sprechen und diese weiter aufzuarbeiten. Auch können Journalist*innen von Betroffenen durchaus Reaktionen erhalten, die von Dankbarkeit für öffentliches Gehör und die Chance zur Selbstermächtigung geprägt sind. B1 erzählte, einer Journalistin für ihre Empathie so dankbar gewesen zu sein, dass er ihr einen Präsentkorb schicken wollte, den diese allerdings aus Gründen der professionellen journalistischen Unabhängigkeit abgelehnt habe. Relevant erscheint, dass mehrere Befragte nach ihrer Medienpräsenz mit anderen Betroffenen in Kontakt kamen - Medienberichte können Betroffenen also offenbar auch dabei helfen, sich vernetzt zu organisieren. B1 gab hierfür folgendes Beispiel: „Andere haben mich schon gefragt: Soll ich an die Öffentlichkeit geben? Und ich habe gesagt: ,Das musst du für dich ganz allein entscheiden'. Für mich waren diese Jabre die härtesten, aber auch die erfülltesten meines Lebens. Und ich mache es auch, weil ich die Power und das Hintergrundwissen habe. Aber ich kenne Betroffene, die nicht mal mehr fähig sind, ibr Leben normal zu gestalten. Aber auch ich merke, der Kampf gebt bei mir ans Eingemachte“.

\section{Fazit}

Unsere Analyse der Perspektive von Betroffenen sexuellen Missbrauchs auf Medien zeigt, dass Journalist*innen bei dieser Thematik eine große Verantwortung gegenüber den Protagonisten ihrer Berichterstattung tragen. Denn die Betroffenen befinden sich vor ihrem Gang in die Öffentlichkeit in einer relativ schwachen Position gegenüber Journalist*innen: Oftmals mit psychischen Problemen beladen, stellen sie meist fest, dass Täter Strafrecht und Kirche kaum (mehr) fürchten müssen. Sie befinden sich daher in Abhängigkeit von einer Medienöffentlichkeit, von der sie sich im Sinne von Selbstermächtigung erhoffen, Kontrolle zurückzuerlangen, indem es gelingt, durch politischen Druck Wiederholungstaten zu verhindern und persönlich die Anerkennung eigenen Leids zu erfahren. 
Erfolgt aus diesen Motiven der Schritt in die Öffentlichkeit, sehen sich Betroffene jedoch häufig mit Journalist*innen konfrontiert, die in der Interaktion mit ihnen einer Medienlogik folgen, welche die Situation der - oftmals traumatisierten und nur unter psychischer Anstrengung sprechfähigen - Betroffenen tendenziell ignoriert. Zu den aus ihrer Sicht besonders problematischen journalistischen Interaktionsmustern gehören wiederholte, sensationalistisch anmutende Fragen nach intimen Details der Straftaten, aber auch Versuche, Bilder von Opfern an Tatorten zu produzieren. Hier wird deutlich, wie wichtig es für Journalist*innen ist, sich in die - äußerlich nicht sichtbare - psychische Situation der Betroffenen hineinzuversetzen und ihrer Anfälligkeit für Re-Traumatisierung Rechnung zu tragen (Maercker \& Mehr 2006). Ein journalistisches Handeln, durch das sich Betroffene in ihrer Autonomie eingeschränkt und bedrängt fühlen, kann ihnen den im Rahmen des Missbrauchs erfahrenen Kontrollverlust unter Umständen salient machen. Auch wenn dieser Effekt von Journalist*innen nicht intendiert ist, sollte er im Sinne einer professionellen Verantwortungsethik bei dieser sensiblen Thematik stets reflektiert werden - gerade auch, weil Journalist*innen diesen Effekt vermutlich nicht selbst feststellen können. Die Befunde haben außerdem gezeigt, dass es für Betroffene überaus belastend sein kann, wenn Journalist*innen über sie in einer Art und Weise berichten, die ihre Integrität und Glaubwürdigkeit infrage stellt. Die allgemeine Norm, wonach Journalist*innen eine kritische Distanz zum Gegenstand ihrer Berichterstattung halten sollen (Baugut 2017), sollte nicht so umgesetzt werden, dass die Missbrauchserfahrungen Betroffener in Medienberichten öffentlich angezweifelt werden.

Vor diesem Hintergrund liegt nahe, dass Betroffene die Möglichkeiten zur Vernetzung in der Online-Welt nutzen, um jene Journalist*innen sorgfältig auszuwählen, mit denen die Interaktion für sie kein gesundheitliches Risiko darstellt. Insgesamt unterstreichen die Befunde zur Interaktion von Betroffenen mit Journalist*innen das Qualitätskriterium, Opfern mit einem hohen Maß an Respekt und Sensibilität zu begegnen (Döring \& Walter 2019). Da diese Ebene der Interaktion der Berichterstattung vorgelagert ist, können Betroffene hier schon feststellen, inwieweit sie eine von Sensationalismus sowie von episodischem Framing geprägte Berichterstattung zu befürchten haben. Beide Berichterstattungsmerkmale widersprechen dem, was journalistische Leitlinien, Betroffene sowie Beratungsstellen für Qualitätskriterien halten: eine auf strukturelle Ursachen von SKM fokussierte Berichterstattung, die sich durch Objektivität und Orientierung an Fakten auszeichnet (ebd.).

Auch wenn sich der Kontakt zu Medienvertretern überaus anstrengend gestalten kann, hält dies manche Betroffene offenbar nicht von ihrem Weg in die Medienöffentlichkeit ab. Ein wichtiger Grund hierfür scheinen relativ starke Medienwirkungsannahmen zu sein. So wird den Medien nicht zuletzt das Potenzial zugeschrieben, andere Betroffene in ihrer Aufarbeitung des Erlebten zu unterstützen. Als einflussreich werden die Medien auch wahrgenommen, wenn es darum geht, auf kirchlicher Seite ernst genommen zu werden - allzu drastische Medienberichterstattung kann dabei jedoch dazu führen, dass Betroffene sich von der Bevölkerung auf diese Rolle reduziert sehen. Obgleich sich Betroffene sexuellen Missbrauchs von Medienöffentlichkeit viel versprechen, sollte also auf journalistischer Seite nicht übersehen werden, dass Betroffene auch Medienwirkungen für möglich halten, die ihnen Angst machen.

Aus Perspektive der Betroffenen können erhoffte und befürchtete Medienwirkungen gleichermaßen eintreten. Einerseits stellen Betroffene fest, dass sie mit ihren Interessen bei der Kirche nur dank Medienöffentlichkeit Gehör finden und dass gerade ihr engeres soziales Umfeld mit Anerkennung und Bewunderung reagiert - zumindest auf journalistische Medieninhalte, bei denen das Unterhaltungsbedürfnis nicht im Mittelpunkt 
steht. Andererseits sind nach Wahrnehmung der Betroffenen aber auch Anfeindungen aus (kirchennahen Teilen) der Bevölkerung nicht ausgeblieben, so dass sich der Weg in die Öffentlichkeit als zweischneidiges Schwert erweisen kann. Entsprechend erleben viele Betroffene Berichterstattung über ihre Fälle als emotional belastend. Dabei sollte nicht übersehen werden, dass Medienaufmerksamkeit für Betroffene, die in vielen Fällen jahrelang um die Anerkennung ihres Leids kämpfen, auch die Gefahr in sich birgt, dass der Wegfall dieser als heilsam empfundenen Aufmerksamkeit negative psychische Folgen haben kann. Doch kontinuierliche Aufmerksamkeit können Medien infolge ihrer wellenartigen Berichterstattung über Skandale wie Missbrauchsfälle nur begrenzt bieten - was darauf hindeutet, dass es hilfreich für Betroffene sein könnte, wenn sie auf ihrem Weg in die Öffentlichkeit psychotherapeutisch begleitet werden. Insgesamt zeigt sich auf Betroffenen-Seite ein differenziertes, realistisches, mitunter ernüchterndes Medienbild, das von Journalist*innen geprägt ist, denen ein Mangel an Reflexion der potenziellen Wirkungen ihrer Berichterstattung auf die Betroffenen attestiert wird. Gerade Lokalmedien werden bisweilen negativ - weil mit kirchlichen Strukturen verflochten wahrgenommen.

Vor diesem Hintergrund verwundert nicht, dass Betroffene auf Berichterstattung auch auf Verhaltensebene intensiv reagieren. Dabei wird im Sinne des Modells reziproker Effekte in der erneuten Interaktion mit Journalist*innen versucht, bestimmte Berichterstattungsmuster zu korrigieren oder zu bestärken. Auch die sorgsame Selektion von Journalist*innen hat sich nicht nur als Ursache, sondern auch als Folge von Berichterstattung erwiesen. Dabei bietet der Online-Bereich den Betroffenen die Möglichkeit zu einem Erfahrungsaustausch, der sie vor Enttäuschungen oder gar Verletzungen infolge journalistischen Verhaltens schützt.

Im Ergebnis erscheint der Weg der Betroffenen sexuellen Missbrauchs in die Medienöffentlichkeit als einer, der aus Betroffenen-Perspektive zwar mit Risiken und emotionaler Belastung verbunden ist, im Sinne der Aufarbeitung und Bekämpfung sexuellen Missbrauchs als ultima ratio aber notwendig erscheint. Journalist*innen stehen vor der Herausforderung, diesen Weg im Rahmen ihrer professionellen Rolle zu unterstützen und dabei zu reflektieren, dass der Themenkomplex des sexuellen Missbrauchs kein gewöhnlicher Gegenstand der Berichterstattung ist.

$\mathrm{Zu}$ den Einschränkungen dieser Studie gehört die Fallzahl dieses (allerdings schwer erreichbaren) Personenkreises, über dessen Medienperspektive bislang kaum Befunde vorlagen. Obwohl wir Hinweise für eine theoretische Sättigung in Bezug auf die uns interessierenden Aspekte von Medienerfahrungen gefunden haben, bleibt eine solche Einschätzung im Rahmen qualitativer Befragungen angesichts relativ niedriger Fallzahlen immer mit einer gewissen Unsicherheit behaftet.

Darüber hinaus ist einschränkend anzumerken, dass die Betroffenen in den Interviews nur über jene Dinge sprechen konnten, die ihnen bewusst bzw. in Erinnerung waren. Nicht ausgeschlossen werden können daher Erinnerungslücken, Wahrnehmungsverzerrungen und Probleme, belastende Ereignisse zu verbalisieren. Gleichwohl deutet das hohe Reflexionsniveau der Befragten darauf hin, dass es im Rahmen dieser Studie gelungen ist, die Perspektive von Betroffenen sexuellen Missbrauchs auf Journalist*innen und deren Berichterstattung erstmals schärfer zu konturieren. 


\section{Literatur}

Allport, G. W. (1954). The Nature of Prejudice. New York: Addison.

Baugut, P. (2017). Politiker und Journalisten in Interaktion. Einfluss des Medienwettbewerbs auf lokale politische Kommunikationskulturen. Wiesbaden: Springer VS. doi: 10.1007/978-3658-15368-7.

Bohrmann, T. (2010). Mediale Gewaltdarstellungen. In C. Schicha \& C. Brosda (Hrsg.), Handbuch Medienethik (S. 417-423). Wiesbaden: Springer VS. doi: 10.1007/978-3-531-92248-5_27.

Brosda, C. (2010). Journalismus. In C. Schicha, C. Brosda (Hg.), Handbuch Medienethik (S. 257277). Wiesbaden. Springer VS. Doi: 10.1007/978-3-531-92248-5_16.

Cromer, L. D., \& Goldsmith, R. E. (2010). Child Sexual Abuse Myths: Attitudes, Beliefs, and Individual Differences. Journal of Child Sexual Abuse, 19(6), 618-647.

Daschmann, G. (2007). Der Preis der Prominenz. Medienpsychologische Überlegungen zu den Wirkungen von Medienberichterstattung auf die dargestellten Akteure. In T. Schierl (Hrsg.), Prominenz in den Medien. Zur Genese und Verwertung von Prominenten in Sport, Wirtschaft und Kultur (S.184-211). Köln: Herbert von Halem Verlag.

Davison, W. P. (1983). The Third-Person Effect in Communication. Public Opinion Quarterly, 47, 1-15.

Döring, N. (2018). Wie wird das Problem des sexuellen Kindesmissbrauchs auf YouTube thematisiert? Zeitschrift für Sexualforschung, 31(04), 333-356.

Döring, N., \& Walter, R. (2019). Media Coverage of Child Sexual Abuse: A Framework of IssueSpecific Quality Criteria. Journal of Child Sexual Abuse. Advance online publication. doi: 10.1080/10538712.2019.1675841.

Dreßing, H., Dölling, D., Hermann, D., Horten, B., Hoell, A., Voss, E., \& Salize, H. J. (2019). Sexueller Missbrauch von Kindern durch katholische Priester seit 2009: Verlauf und relative Häufigkeit im Vergleich zur männlichen Allgemeinbevölkerung. Psychiat Prax, 46, 1-7. doi: 10.1055/a-0936-3869.

Dreßing, H., Salize, H. J., Dölling, D., Hermann, D., Kruse, A., Schmitt, E., \& Bannenbger, B. (2018). Sexueller Missbrauch an Minderjährigen durch katholische Priester, Diakone und männliche Ordensangehörige im Bereich der Deutschen Bischofskonferenz. https://www.dbk.de/file admin/redaktion/diverse_downloads/dossiers_2018/MHG-Studie-gesamt.pdf [14.09.2020].

Füller, C. (2011). Sündenfall: Wie die Reformschule ibre Ideale missbrauchte. Köln: DuMont Buchverlag.

Fusch, P. I., \& Ness, L. R. (2015). Are We There Yet? Data Saturation in Qualitative Research. The Qualitative Report, 20(9), 1408-1416.

Fritsche, I., Jonas, E., \& Kessler, T. (2011). Collective Reactions to Threat: Implications for Intergroup Conflict and for Solving Societal Crises. Social Issues and Policy Review, 5, 101-136. doi: 10.1111/j.1751-2409.2011.01027.x.

Gunther, A. C. (1998). The Persuasive Press Inference Effects of Mass Media on Perceived Public Opinion. Communication Research, 25, 486-504.

Gunther, A. C., \& Storey, J. D. (2003). The Influence of Presumed Influence. Journal of Communication, 53, 199-215.

Hanitzsch, T., Steindl, N., \& Lauerer, C. (2016). Country Report: Journalists in Germany. Worlds of Journalism Study, urn:nbn:de:bvb:19-epub-28095-3.

Herman, J. (2018). Die Narben der Gewalt: Traumatische Erfabrungen verstehen und überwinden [5. Auflage]. Paderborn: Junfermann Verlag.

Hinkelman, L., \& Bruno, M. (2008). Identification and Reporting of Child Sexual Abuse: The Role of Elementary School Professionals. The Elementary School Journal, 108(5), 376-391.

Hölling, I. (2007). Empfehlungen für die Berichterstattung über sexuelle Gewalt. Im Mittelpunkt: Sensibler Umgang mit Frauen und Mädchen, die sexuelle Gewalt erlebt haben. In Wildwasser e. V. Berlin (Hrsg.), Respekt und Würde: Sexuelle Gewalt als Thema in den Medien (S.143147). Bonn: Mebes \& Noack.

Hopf, C. (2008). Qualitative Interviews - ein Überblick. In U. Flick, E. von Kardoff \& I. Steinke (Hrsg.), Qualitative Forschung. Ein Handbuch (S. 349-360). Reinbek: Rowohlt-Taschenbuch Verlag. 
Hove, T., Paek, H. J., Isaacson, T., \& Cole, R. T. (2013). Newspaper Portrayals of Child Abuse: Frequency of Coverage and Frames of the Issue. Mass Communication and Society, 16(1), 89108.

Johnson, J. M. (2017). Horror Stories and the Construction of Child Abuse. In J. Best (Hrsg.), Images of Issues. Typifying Contemporary Social Problems (pp. 17-32). London/ New York: Routledge.

Kavemann, B., Graf-van Kesteren, A., Rothkegel, S., \& Nagel, B. (2016). Erinnern, Schweigen und Sprechen nach sexueller Gewalt in der Kindheit: Ergebnisse einer Interviewstudie mit Franen und Männern, die als Kind sexuelle Gewalt erlebt haben. Wiesbaden: Springer VS. doi: 10.1007/978-3-658-10510-5.

Kavemann, B., Nagel, B., Doll, D., \& Helfferich, C. (2018). Erwartungen Betroffener sexuellen Kindesmissbrauchs an gesellschaftliche Aufarbeitung. https://www.aufarbeitungskommissi on.de/wp-content/uploads/2019/09/Studie_Erwartungen-Betroffener-sexuellen-Kindesmiss brauchs-an-die-gesellschaftliche-Aufarbeitung.pdf [14.09.2020].

Kepplinger, H. M. (2007). Reciprocal Effects: Toward a Theory of Mass Media Effects on Decision Makers. Harvard International Journal of Press/Politics, 12, 3-23.

Kepplinger, H. M. (2009). Publizistische Konflikte und Skandale. Wiesbaden: Springer VS.

Kepplinger, H. M. (2010). Medieneffekte. Wiesbaden: Springer VS.

Kepplinger, H. M., \& Glaab, S. (2005). Folgen ungewollter Öffentlichkeit: Abwertende Pressebeiträge aus der Sichtweise der Betroffenen. In A. Beater \& S. Habermeier (Hrsg.), Verletzung von Persönlichkeitsrechten durch die Medien (S. 117-137). Tübingen: Verlag Mohr Siebeck.

Kepplinger, H. M., \& Marx, D. (2008). Wirkungen und Rückwirkungen der politischen Kommunikation. Reziproke Effekte auf Landtagsabgeordnete. In U. Sarcinelli \& J. Tenscher (Hrsg.), Politikherstellung und Politikdarstellung. Beiträge zurpolitischen Kommunikation (S. 188-208). Köln: Herbert von Halem Verlag.

Kepplinger, H. M., \& Zerback, T. (2009). Der Einfluss der Medien auf Richter und Staatsanwälte. Publizistik, 54, 216-239.

Kitzinger, J. (2004). Framing Abuse: Media Influence and Public Understanding of Sexual Violence against Children. London: Pluto Press.

Kunczik, M., \& Bleh, W. (1995). Kriminalitätsopfer in der Zeitungsberichterstattung. Folgen der Berichterstattung aus der Perspektive der Opfer. Mainz: Weißer Ring.

Lamnek, S. (2005). Qualitative Sozialforschung. [vollständig überarbeitete Auflage]. Weinheim, Basel: Beltz.

Lang, K., \& Lang, G. E. (1953). The Unique Perspective of Television and its Effect: A Pilot Study. American Sociological Review, 18, 3-12. doi: 10.1007/BF02713365.

Maercker, A., \& Mehr, A. (2006). What if Victims Read a Newspaper Report About Their Victimization? A Study on the Relationship to PTSD Symptoms in Crime Victims. European Psychologist, 11, 137-142. doi: 10.1027/1016-9040.11.2.137.

Martinius, J. (2009). Macht der Medien und ihrer Informanten. Monatsschr Kinderheilkd, 157, 456460. doi: 10.1007/s00112-008-1933-2.

Mathews, B., Collin-Vézina, D. (2016). Child Sexual Abuse: Raising Awareness and Empathy is Essential to Promote New Public Health Responses. J Public Health Pol, 37, 304-314. doi: 10.1057/jphp.2016.21.

Maydell, E. (2018). 'It just Seemed Like Your Normal Domestic Violence': Ethnic Stereotypes in Print Media Coverage of Child Abuse in New Zealand. Media, Culture E Society, 40(5), 707724. doi: 10.1177/0163443717737610.

Mayring, P. (2015). Qualitative Inhaltsanalyse. Grundlagen und Techniken. Weinheim und Basel: Beltz.

Mejia, P., Cheyne, A., \& Dorfman, L. (2012). News Coverage of Child Sexual Abuse and Prevention, 2007-2009. Journal of Child Sexual Abuse: Research, Treatment, \& Program Innovations for Victims, Survivors, \& Offenders, 21(4), 470-487. doi: 10.1080/10538712.2012.692465.

Meyen, M., \& Riesmeyer, C. (2009). Diktatur des Publikums. Journalisten in Deutschland. Konstanz: UVK.

Müller, N., \& Nicolai, E. M. (2007). Zwischen gelungener und misslungener Kooperation: Unterschiedliche Sichtweisen auf die Zusammenarbeit mit einer Zeitung. In Wildwasser e. V. Berlin 
(Hrsg.), Respekt und Würde: Sexuelle Gewalt als Thema in den Medien (S.111-118). Bonn: Mebes \& Noack.

Niner, S., Ahmad, Y., \& Cuthbert, D. (2013). The 'Social Tsunami': Media Coverage of Child Abuse in Malaysia's English-language Newspapers in 2010. Media, Culture E Society, 35(4), 435-453.

Popović, S. (2018). Child Sexual Abuse News: A Systematic Review of Content Analysis Studies. Journal of Child Sexual Abuse, 27(7), 752-777. doi:0.1080/10538712.2018. 1486935.

Rörig, J. W. (2017). Schweigen hilft Tätern und Täterinnen. Was für einen verbesserten Schutz vor sexuellem Missbrauch getan werden kann. BdW Blätter der Woblfabrtspflege, 164, 59-62. doi: 10.5771/0340-8574-2017-2-59.

Scheufele, B. (2005). Sexueller Missbrauch: Mediendarstellung und Medienwirkung. Wiesbaden: Springer VS.

Terry, K. J., \& Jay, J. (2015). Child Sexual Abuse within the Catholic Church: A Review of Global Perspectives. International Journal of Comparative and Applied Criminal Justice, 39(2), 139154. doi: 10.1080/01924036.2015.1012703.

Thomaß, B. (2003). Fünf ethische Prinzipien journalistischer Praxis. In B. Debatin \& R. Funiok (Hrsg.), Kommunikations- und Medienethik (S. 159-168). Konstanz: UVK.

Vallone, R. P., Ross, L., \& Lepper, M. R. (1985). The Hostile Media Phenomenon: Biased Perception and Perceptions of Media Bias in Coverage of the Beirut Massacre. Journal of Personality and Social Psychology, 49, 577-585.

Waller, L., Dreher, T., Hess, K., McCallum, K., \& Skogerbø, E. (2020). Media Hierarchies of Attention: News Values and Australia's Royal Commission into Institutional Responses to Child Sexual Abuse. Journalism Studies, 21(2), 180-196. doi: 10.1080/1461670X.2019.1633244.

Weatherred, J. L. (2015). Child Sexual Abuse and the Media: A Literature Review. Journal of Child Sexual Abuse, 24, 16-34. doi: 10.1080/10538712.2015.976302.

Witt, A., Brähler, E., Plener, P. L., \& Fegert, J. M. (2019). Different Contexts of Sexual Abuse with a Special Focus on the Context of Christian Institutions: Results from the General Population in Germany. Journal of Interpersonal Violence, 34, 1-22. doi: 10.1177/0886260519888540.

ZEIT ONLINE (2019). Sexueller Kindesmissbrauch. Was passiert, wenn man spricht. https:// www.zeit.de/2019/30/sexueller-kindesmissbrauch-opfer-bistum-trier-katholische-kirche [14.09.2020]. 